\title{
Frame Dependence of the Pair Contribution to the Pion Electromagnetic Form Factor in a Light-Front Approach
}

\author{
J. P. B. C. de Melo ${ }^{a}$, T. Frederico ${ }^{b}$, E. Pace ${ }^{c}$, and G. Salmè ${ }^{d}$ \\ ${ }^{a}$ Instituto de Física Teórica, \\ Universidade Estadual Paulista, 01405-900, São Paulo, SP, Brazil \\ ${ }^{b}$ Dep. de Física, Instituto Tecnológico da Aeronáutica, \\ Centro Técnico Aeroespacial, 12.228-900, São José dos Campos, São Paulo, Brazil \\ ${ }^{c}$ Dipartimento di Fisica, \\ Università di Roma "Tor Vergata" and Istituto Nazionale di Fisica Nucleare, Sezione Tor Vergata, \\ Via della Ricerca Scientifica 1, I-00133 Roma, Italy \\ ${ }^{d}$ Istituto Nazionale di Fisica Nucleare, \\ Sezione Roma I, P.le A. Moro 2,I-00185 Roma, Italy
}

Received on 30 October, 2002

\begin{abstract}
The frame dependence of the pair-term contribution to the electromagnetic form factor of the pion is studied within the Light Front approach. A symmetric ansatz for the pion Bethe-Salpeter amplitude with a pseudo scalar coupling of the constituent to the pion field is used. In this model, the pair term vanishes for the DrellYan condition, while it is dominant for momentum transfer along the light-front direction.
\end{abstract}

\section{Introduction}

Within the Front Form dynamics[1], where the state of the system is defined at $x^{+}=t+z=0$, if one uses the impulse approximation of the plus component of the electromagnetic current $\left(j^{+}\right)$in the Drell-Yan frame to calculate the form factors, the pair production from the incoming photon (pair-term contribution) is in general suppressed by lightfront momentum conservation (see, e.g., [2]). This was seen in schematic covariant models for spin-zero composite systems $[3,4,5]$. However, even in the Drell-Yan frame the pair term is present in $j^{+}$for spin-one systems and is necessary to keep the rotational properties of the matrix element of the current[6, 7].

To avoid the difficulties associated with the rotational properties of the impulse approximation some physically motivated schemes to extract form factors from the current were used[8, 9, 10]. In another approach, free of these ambiguities[11], the plus component of the momentum transfer is non zero while the transverse momentum transfer vanishes in the Breit frame. This can be achieved by departing from the Drell-Yan condition by rotating the system around the $y$-direction, i.e, a non-kinematical transformation, and thus changing the direction of the momentum transfer in the $z-x$ plane.

However, by relaxing the Drell-Yan condition, a lightfront pair term can contribute to the plus component of the current, which can be studied in the pion example, as a pro- totype of a relativistic system of bound constituents. In this work, the composite system of a constituent quark and antiquark, is described by an ansatz for the Bethe-Salpeter amplitude which is nonconstant and symmetric with a pseudo scalar coupling of the constituent to the pion field. Our aim here is to discuss, within that model, the magnitude of the pair contribution to the pion electromagnetic form factor for momentum transfers in the $z-x$ plane in the Breit-frame, as has been done in Ref.[5].

The work is organized as follows. In Sec. II, we present the model of the pion Bethe-Salpeter amplitude and its eletromagnetic current in impulse approximation. In Sec. III, we discuss the numerical results for the pion electromagnetic form factor where the separate contribution of the pair term is given. We also present our summary in Sec. III.

\section{Pion Model and Electromagnetic Current}

The electromagnetic current of the pion is calculated in impulse approximation, using a pseudoscalar coupling between pion and quark fields, given by the effective Lagrangian (see, e.g. [12]):

$$
\mathcal{L}_{I}=-\imath g \vec{\Phi} \cdot \bar{q} \gamma^{5} \vec{\tau} q
$$

where $g=m / f_{\pi}$ is the coupling constant from the Goldberg-Treiman relation at the quark level, and $m$ is the 
mass of the constituents and $f_{\pi}$ the pion decay constant.

The electromagnetic current of $\pi^{+}$in impulse approximation is build from the covariant expression, which correspond to the Feynman triangle diagram (see, e.g., [13]):

$$
\begin{aligned}
j^{\mu} & =-\imath 2 e \frac{m^{2}}{f_{\pi}^{2}} N_{c} \int \frac{d^{4} k}{(2 \pi)^{4}} \Lambda\left(k, P^{\prime}\right) \Lambda(k, P) \\
& \times \operatorname{Tr}\left[S(k) \gamma^{5} S\left(k-P^{\prime}\right) \gamma^{\mu} S(k-P) \gamma^{5}\right],
\end{aligned}
$$

where $S(p)=\frac{1}{p-m+\imath \epsilon}, N_{c}=3$ is the number of colors, $P^{\mu}$ and $P^{\prime \mu}=P^{\mu}+q^{\mu}$ are the initial and final momenta of the system, $q^{\mu}$ is the momentum transfer and $k^{\mu}$ the spectator quark momentum. The factor 2 stems from isospin algebra.

Our ansatz for the analytical form of the vertex function describing the momentum part of the coupling between the constituents and pion is:

$$
\Lambda(k, P)=\frac{C}{\left(k^{2}-m_{R}^{2}+\imath \epsilon\right)}+\frac{C}{\left((P-k)^{2}-m_{R}^{2}+\imath \epsilon\right)},
$$

where $m_{R}$ is the regulator parameter. By imposing the charge normalization condition $F_{\pi}\left(q^{2}=0\right)=1$, the constant $C$ is fixed. This model satisfies current conservation $q \cdot j=0[5]$.

We consider Breit frames, with the momentum transfer $q^{+} \neq 0$ and using the light-front variables, i.e. $k^{+}=$ $k^{0}+k^{3}, k^{-}=k^{0}-k^{3}, \vec{k}_{\perp} \equiv\left(k^{1}, k^{2}\right)$, one has

$q^{+}=-q^{-}=\sqrt{-q^{2}} \sin \alpha, q_{x}=\sqrt{-q^{2}} \cos \alpha, q_{y}=0$,

and $q^{2}=q^{+} q^{-}-\left(\vec{q}_{\perp}\right)^{2}$. The Drell-Yan frame with $q^{+}=0$ is recovered for $\alpha=0$, while the $q^{+}=\sqrt{-q^{2}}$ condition [11] comes with $\alpha=90^{\circ}$. (The angle $\theta$ of Ref.[14] corresponds to $\alpha+90^{\circ}$ ).

The pion electromagnetic form factor is extracted from the general covariant expression:

$$
j^{\mu}=e\left(P^{\mu}+P^{\prime \mu}\right) F_{\pi}\left(q^{2}\right)
$$

evaluating the plus component of the current in Eq.(2), which has two non vanishing contributions: $[3,5,14,15]$ :

$$
F_{\pi}\left(q^{2}\right)=F_{\pi}^{(I)}\left(q^{2}, \alpha\right)+F_{\pi}^{(I I)}\left(q^{2}, \alpha\right)
$$

The integration over the interval of $0 \leq k^{+}<P^{+}$defines $F_{\pi}^{(I)}\left(q^{2}, \alpha\right)$ (see Fig. 1(a)). For $k^{+}$in the integration interval $P^{+} \leq k^{+} \leq P^{\prime}$ (see Fig. 1(b)) one defines $F_{\pi}^{(I I)}\left(q^{2}, \alpha\right)$. In this model the contribution of the valence component of the wave function is part of $F_{\pi}^{(I)}\left(q^{2}, \alpha\right)$. The pair term contribution to the form factor, with $q^{+}>0$, is $F_{\pi}^{(I I)}\left(q^{2}, \alpha\right)$.

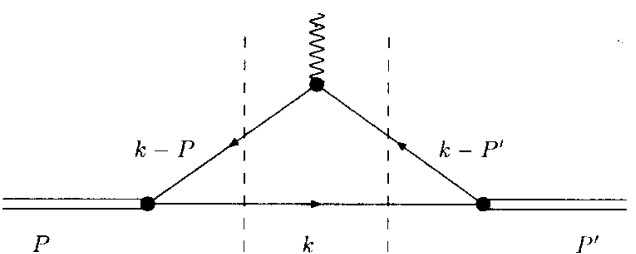

(a)

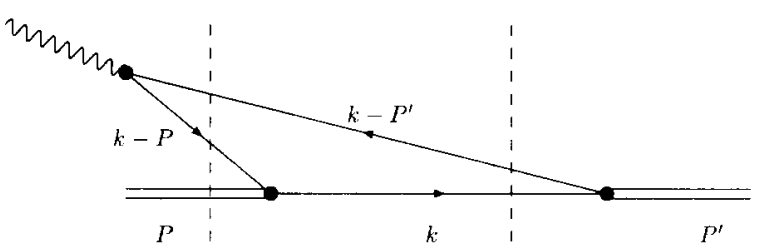

(b)

Figure 1. Light-front time-ordered diagrams for the current: (a) $F_{\pi}^{(I)}$ (Eq.(7)) and (b) $F_{\pi}^{(I I)}$ (Eq. (8)).

The two contributions to the form factor are given by:

$$
\begin{aligned}
F_{\pi}^{(I)}\left(q^{2}, \alpha\right) & =-\imath \frac{m^{2}}{\left(P^{+}+P^{\prime+}\right) f_{\pi}^{2}} \frac{N_{c}}{(2 \pi)^{4}} \int d^{2} k_{\perp} d k^{+} d k^{-} \\
& \times \frac{\theta\left(k^{+}\right) \theta\left(P^{+}-k^{+}\right)}{k^{+}\left(P^{+}-k^{+}\right)\left(P^{\prime}-k^{+}\right)} \Pi\left(k, P, P^{\prime}\right)(7)
\end{aligned}
$$

and

$$
\begin{aligned}
F_{\pi}^{(I I)}\left(q^{2}, \alpha\right) & =-\imath \frac{m^{2}}{\left(P^{+}+P^{\prime+}\right) f_{\pi}^{2}} \frac{N_{c}}{(2 \pi)^{4}} \int d^{2} k_{\perp} d k^{+} d k^{-} \\
& \times \frac{\theta\left(k^{+}-P^{+}\right) \theta\left(P^{\prime+}-k^{+}\right)}{k^{+}\left(P^{+}-k^{+}\right)\left(P^{\prime}-k^{+}\right)} \Pi\left(k, P, P^{\prime}\right)(8)
\end{aligned}
$$

where

$$
\begin{gathered}
\Pi\left(k, P, P^{\prime}\right)=\frac{\operatorname{Tr}\left[\mathcal{O}^{+}\right] \Lambda(k, P) \Lambda\left(k, P^{\prime}\right)}{\left(P^{-}-k^{-}-(P-k)_{o n}^{-}+\frac{\imath \epsilon}{P^{+}-k^{+}}\right)} \\
\times \quad \frac{1}{\left(k^{-}-k_{o n}^{-}+\imath \epsilon\right)\left(P^{\prime-}-k^{-}-\left(P^{\prime}-k\right)_{o n}^{-}+\imath \epsilon\right)}(9)
\end{gathered}
$$

with,

$$
\mathcal{O}^{+}=(k+m) \gamma^{5}\left(k-\mathbb{P}^{\prime}+m\right) \gamma^{+}(k-\mathbb{P}+m) \gamma^{5} \text {. }
$$

The suffix on indicates particles on- $k^{-}$-shell. In particular, for the Drell-Yan condition $\left(q^{+}=0\right) F_{\pi}^{(I I)}\left(q^{2}, \alpha\right)$ vanishes.

The Dirac propagator written in terms of light-front momenta has two parts [16]:

$$
\frac{k+m}{k^{2}-m^{2}+\imath \epsilon}=\frac{k_{o n}+m}{k^{+}\left(k^{-}-k_{o n}^{-}+\frac{\imath \epsilon}{k^{+}}\right)}+\frac{\gamma^{+}}{2 k^{+}},
$$

where $k_{\text {on }}^{-}=\left(k_{\perp}^{2}+m^{2}\right) / k^{+}$. In the right-hand side of Eq.(11), the first term is propagating in the light-front time and the second one is instantaneous. This second term contributes to both $F_{\pi}^{(I)}\left(q^{2}\right)$ and $F_{\pi}^{(I I)}\left(q^{2}\right)$, due to the analytic structure of the symmetric vertex function of Eq. (3). The contribution of the instantaneous term is of nonvalence nature because it is left out in the definition of the valence wave function, as we discuss below. 
The pion Bethe-Salpeter amplitude within the model is given by:

$$
\begin{aligned}
& \Psi(k, P)= \\
& \frac{m}{f_{\pi}} \frac{k+m}{k^{2}-m^{2}+\imath \epsilon} \gamma^{5} \Lambda(k, P) \frac{k-\mathbb{P}+m}{(k-P)^{2}-m^{2}+\imath \epsilon}(12)
\end{aligned}
$$

from which the momentum component of the valence light-front wave function, $\Phi\left(k^{+}, \vec{k}_{\perp} ; P^{+}, \vec{P}_{\perp}\right)[5]$, is derived eliminating the relative time between the quarks after dropping the instantaneous terms of the external Dirac propagators[17]. Also, the factors containing gamma matrices in the numerator and the phase space factors $k^{+}$and $\left(P^{+}-k^{+}\right)$appearing in the denominator are left out[5], and then one gets:

$$
\begin{aligned}
& \Phi\left(k^{+}, \vec{k}_{\perp} ; P^{+}, \vec{P}_{\perp}\right)=\left[\frac{\mathcal{N}}{(1-x)\left(m_{\pi}^{2}-\mathcal{M}^{2}\left(m^{2}, m_{R}^{2}\right)\right)}\right. \\
& \left.+\frac{\mathcal{N}}{x\left(m_{\pi}^{2}-\mathcal{M}^{2}\left(m_{R}^{2}, m^{2}\right)\right)}\right] \frac{P^{+}}{m_{\pi}^{2}-M_{0}^{2}} .
\end{aligned}
$$

where $\mathcal{N}=\sqrt{N_{c}} C m / f_{\pi}$, is a normalization factor and $x=k^{+} / P^{+}$, with $0 \leq x \leq 1 ; \mathcal{M}^{2}\left(m_{a}^{2}, m_{b}^{2}\right)=$ $\frac{k_{\perp}^{2}+m_{a}^{2}}{x}+\frac{(P-k)_{\perp}^{2}+m_{b}^{2}}{1-x}-P_{\perp}^{2} ;$ and the square of the free mass is $M_{0}^{2}=\mathcal{M}^{2}\left(m^{2}, m^{2}\right)$. The light-front wave function, Eq.(13), is symmetric by the interchange of quark and antiquark momenta, therefore it is not plagued by the conceptual difficulties associated with the use of the nonsymmetric regulator [14].

The electromagnetic form factor evaluated in the Breit frame using only the valence component is given by:

$$
\begin{aligned}
& F_{\pi}^{(W F)}\left(q^{2}, \alpha\right)=\frac{1}{2 \pi^{3}\left(P^{\prime+}+P^{+}\right)} \int d^{2} k_{\perp} \int_{0}^{P^{+}} d k^{+} \\
\times \quad & {\left[k_{\text {on }}^{-} P^{+} P^{\prime+}+\frac{1}{2} \vec{k}_{\perp} \cdot \vec{q}_{\perp}\left(P^{\prime+}-P^{+}\right)-\frac{1}{4} k^{+} q_{\perp}^{2}\right] } \\
\times \quad & \frac{\Phi\left(k^{+}, \vec{k}_{\perp} ; P^{\prime+}, \frac{\vec{q}_{\perp}}{2}\right) \Phi\left(k^{+}, \vec{k}_{\perp} ; P^{+},-\frac{\vec{q}_{\perp}}{2}\right)}{k^{+}\left(P^{+}-k^{+}\right)\left(P^{\prime}+k^{+}\right)},
\end{aligned}
$$

where normalization constant $C$ is determined from the condition $F_{\pi}(0)=1$ in Eq. (6). The probability of the valence component in the pion, $\eta$, is identified to $F_{\pi}^{(W F)}(0,0)$

The pion decay constant is one constraint to fix the free parameters of the model:

$$
P_{\mu}<0\left|A_{i}^{\mu}\right| \pi_{j}>=\imath m_{\pi}^{2} f_{\pi} \delta_{i j},
$$

where $A_{i}^{\mu}=\bar{q} \gamma^{\mu} \gamma^{5} \frac{\tau_{i}}{2} q$ is the isovector axial current. With our ansatz for the pion- $\bar{q} q$ vertex function, one gets

$$
\begin{aligned}
f_{\pi} & =-\imath \frac{m}{f_{\pi}} \frac{N_{c}}{m_{\pi}^{2}} \int \frac{d^{4} k}{(2 \pi)^{4}} \\
& \times \operatorname{Tr}\left[\mathbb{P} \gamma^{5} S(k) \gamma^{5} S(k-P)\right] \Lambda(k, P),
\end{aligned}
$$

and integrating on $k^{-}$, one arrives at

$$
f_{\pi}=\frac{m \sqrt{N_{c}}}{4 \pi^{3}} \int \frac{d^{2} k_{\perp} d k^{+}}{k^{+}\left(m_{\pi}-k^{+}\right)} \Phi\left(k^{+}, \vec{k}_{\perp} ; m_{\pi}, \overrightarrow{0}\right),
$$

expressed in terms of the valence component of the wave function [12].

\section{Numerical Results and Summary}

In this model, we have two free parameters: the constituent quark mass $m$, which is chosen as $0.220 \mathrm{GeV}[12,18,19]$ and the regulator mass, $m_{R}$, found to be $0.6 \mathrm{GeV}$ from the fit of the experimental value $f_{\pi}^{e x p}=92.4 \mathrm{MeV}$. The pion mass used is $0.140 \mathrm{GeV}$. With these parameters, the charge radius from $\left\langle r^{2}\right\rangle=6 \frac{\partial}{\partial q^{2}} F_{\pi}$, comes out to be 0.74 $\mathrm{fm}$, which is about $10 \%$ larger than the experimental value ( $\left.r_{\text {exp }}=0.67 \pm 0.02 \mathrm{fm}[20]\right)$. The probability of the $q \bar{q}$ Fock-state component in the pion in the model is calculated to be $\eta=0.77$, differently from the nonsymmetric regulator model of Ref. [4], where $\eta=1$. We note that, in a previous work[12] it was necessary a probability around $0.5-0.75$ of the valence wave function to fit the data on deep inelastic scattering.

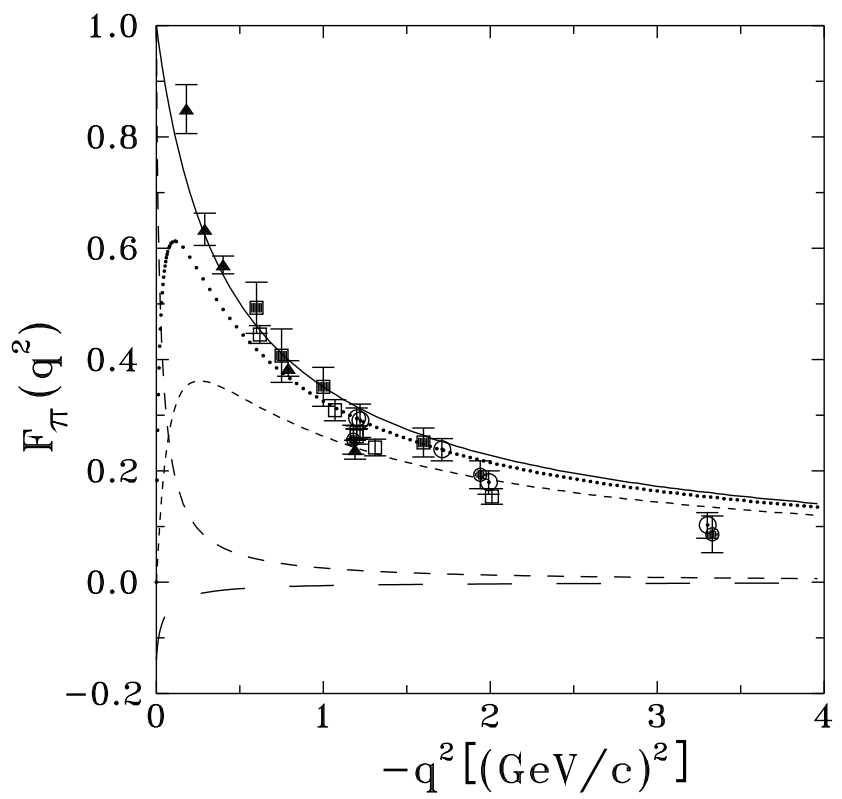

Figure 2. Pion form factor as a function of $-q^{2}$ for $\alpha=$ $90^{\circ}$. Theoretical results: $F_{\pi}\left(q^{2}\right)$ (solid line); $F_{\pi}^{(I)}\left(q^{2}, \alpha\right)$ (dashed line); $F_{\pi}^{(I I)}\left(q^{2}, \alpha\right)$ (dotted line); $F_{\pi \text { inst }}^{(I)}\left(q^{2}, \alpha\right)$ (long-dashed line); $F_{\pi \text { inst }}^{(I I)}\left(q^{2}\right)$ (short-dashed line). Experimental data: Ref. [21] (full squares), Ref. [22] (full triangles), Ref. [23] (empty squares), Ref. [24] (empty circles) and Ref. [25] (full circles).

In Fig. 2, the results for the pion form factor are shown and compared to the experimental data. The full-model calculations, Eq. (6), nicely agree with the new data for the pion form factor [21]. Therefore, our symmetric vertex model can reproduce the form factor data consistently with the experimental value of $f_{\pi}$, while for the nonsymmetric regulator this was not achieved[4]. We observe that the model reproduces simultaneously $f_{\pi}$ and the experimental form factor for constituent quark mass in the range between 0.2 and $0.3 \mathrm{GeV}$. 
The separate contributions to the pion form factor, $F_{\pi}^{(I)}$ and $F_{\pi}^{(I I)}$ for $\alpha=90^{\circ}$, are shown in Fig. 2. Differently from the case $\alpha=0^{\circ}$, the form factor is dominated by the pair production process for $\alpha=90^{\circ}$, except near $q^{2}=0$. Also, we observe that the form factor is completely dominated by the pair-term contribution at high values of the momentum transfer, which appears to be fairly model independent as well[5, 14].

The contributions of the instantaneous part of the Dirac propagator to $F_{\pi}^{(I)}\left(q^{2}, \alpha\right)$ and $F_{\pi}^{(I I)}\left(q^{2}, \alpha\right)$, called $F_{\pi \text { inst }}^{(I)}\left(q^{2}, \alpha\right)$ and $F_{\pi \text { inst }}^{(I I)}\left(q^{2}, \alpha\right)$, respectively, are also shown in Fig. 2 for $\alpha=90^{\circ}$. The value of $F_{\pi \text { inst }}^{(I)}\left(q^{2}, \alpha\right)$ is nonzero because of the specific analytic structure of the vertex function[5]. We also observe that $F_{\pi \text { inst }}^{(I I)}\left(q^{2}, \alpha\right)$ dominates $F_{\pi}\left(q^{2}\right)$ at higher momentum transfers. One can understand this by looking at the diagram of Fig. 1(b), where in principle the spectator quark can be exchanged between the incoming and outgoing pion at a given instant $x^{+}$, while the quark-antiquark pair has been produced by the virtual photon at an earlier stage. As the magnitude of the momentum $q^{-}\left(=-q^{+}\right)$increases, the time fluctuation for the virtual process decreases and favors the instantaneous exchange of the spectator quark between the initial and final pion, which finally explains the dominance of $F_{\pi \text { inst }}^{(I I)}\left(q^{2}, \alpha\right)$ in the pion form factor. In Fig. 3, the results for the various contributions to the pion form factor for $-q^{2}=1(\mathrm{GeV} / \mathrm{c})^{2}$ as a function of the angle $\alpha$ are shown. For increasing angles, the form factor changes smoothly from valence to pair-term or nonvalence dominance.

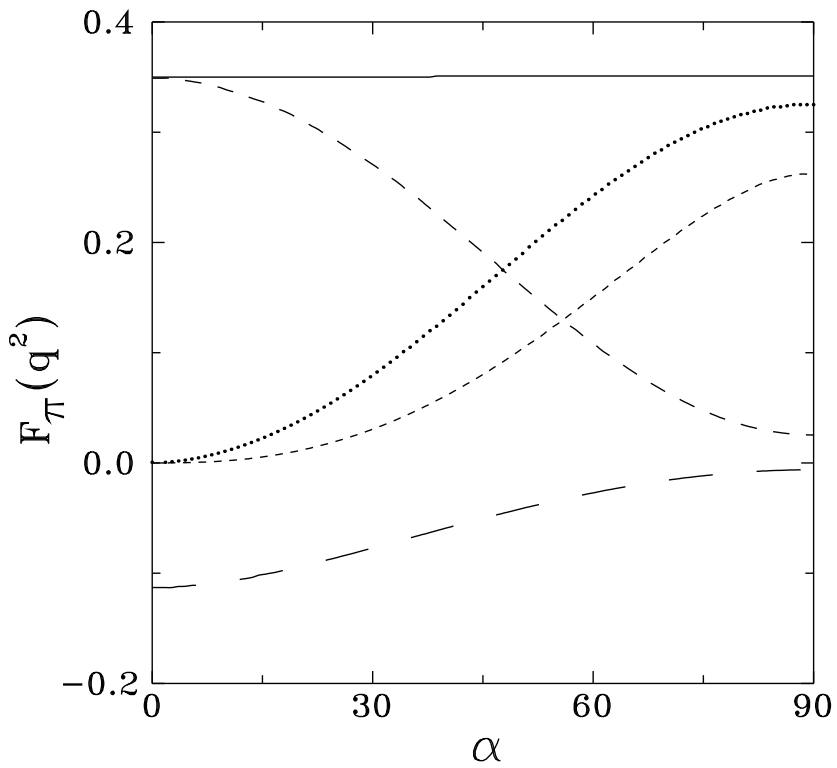

Figure 3. Contributions to the pion form factor vs $\alpha$ for $-q^{2}=$ $1(\mathrm{GeV} / \mathrm{c})^{2}$. Theoretical results: $F_{\pi}\left(q^{2}\right)$ (solid line); $F_{\pi}^{(I)}\left(q^{2}, \alpha\right)$ (dashed line) ; $F_{\pi}^{(I I)}\left(q^{2}, \alpha\right)$ (dotted line); $F_{\pi \text { inst }}^{(I)}\left(q^{2}, \alpha\right)$ (longdashed line); $F_{\pi \text { inst }}^{(I I)}\left(q^{2}, \alpha\right)$ (short-dashed line).

In summary, we verified that the new data for the pion electromagnetic form factor[21] is satisfactory described by our symmetric ansatz of the vertex function when the experimental value of $f_{\pi}^{e x p}$ is fitted and the constituent quark mass is chosen in the range between 0.2 and $0.3 \mathrm{GeV}$. We performed a detailed analysis of the contribution of the lightfront pair-term to the form factor for different momentum transfer directions in the Breit frame. Such contribution is unique and does not depend on the particular choice of Bethe-Salpeter vertex, as long as the four-dimensional impulse approximation is used to calculate the electromagnetic current. Another interesting outcome of our symmetric model is that the probability of the pion valence component, $\eta$, is about 0.77 ), at variance with previous covariant calculations where $\eta=1[4,14]$.

We thank the funding agencies FAPESP (Fundação de Amparo a Pesquisa do Estado de São Paulo), and CNPq (Conselho Nacional de Pesquisa e Desenvolvimento), and Ministero della Ricerca Scientifica e Tecnologica for partial support.

\section{References}

[1] P. A. M. Dirac, Rev. Mod. Phys. 21, 392 (1949).

[2] L.L. Frankfurt and M.I. Strikman, Nucl. Phys. B148, 107 (1979).

[3] M. Sawicki, Phys. Rev. D44, 433 (1991); Phys. Rev. D46, 474 (1992).

[4] J. P. B. C. de Melo, H. W. Naus and T. Frederico, Phys. Rev. C59, 2278 (1999).

[5] J. P. B. C. de Melo, T. Frederico, E. Pace and G. Salmè, Nucl. Phys. A707, 399 (2002).

[6] J. P. B. C. de Melo, J. H. O. Sales, T. Frederico and P. U. Sauer, Nucl. Phys. A631, 574c (1998); J. P. B. C. de Melo, T. Frederico, H. W. L. Naus and P. U. Sauer, Nucl. Phys. A660, 219 (1999).

[7] B.L.G. Bakker, H.-M. Choi and C.-R. Ji, Phys. Rev. D65, 116001 (2002).

[8] I. L. Grach and L. A. Kondratyuk, Sov. J. Nucl. Phys. 39, 198 (1984).

[9] L. L. Frankfurt, T. Frederico and M. I. Strikman, Phys. Rev. C48, 2182 (1993).

[10] J. Carbonell, B. Deplanques, V. A. Karmanov and J.-F. Mathiot, Phys. Rep. 300, 215 (1998).

[11] F. M. Lev, E. Pace and G. Salmè, Nucl. Phys. A641, 229 (1998); Few-Body Syst. Suppl. 10, 135 (1998); Phys. Rev. Lett. 83, 5250 (1999); Phys. Rev. C62, 064004 (2000); Nucl. Phys. A663, 365 (2000); E. Pace and G. Salmè, Nucl. Phys. A684, 487 (2001); A689, 411 (2001).

[12] T. Frederico and G. A. Miller, Phys. Rev. D45, 4207 (1992); Phys. Rev. D50, 210 (1994).

[13] S.J. Brodsky and J. R. Primack, Ann. Phys. 52, 315 (1969), and references therein quoted; F. Coester and D.O. Riska, Ann. Phys. 234, 141 (1994).

[14] B. L. G. Bakker, H.-M. Choi and C.-R. Ji, Phys. Rev. D63, 074014 (2001).

[15] H.-M. Choi and C.-R. Ji, Phys. Rev. D58, 071901 (1998); Phys. Rev. D59, 034001 (1999). 
[16] S. J. Brodsky, H. C. Pauli, and S. S. Pinsky, Phys. Rep. 301, 299 (1998)

[17] J. H. O. Sales, T. Frederico, B. V. Carlson and P. U. Sauer, Phys. Rev. C63, 064003 (2001).

[18] S. Godfrey and N. Isgur, Phys. Rev. D32, 185 (1985).

[19] F. Cardarelli, I.L. Grach, I.M. Narodetskii, E. Pace, G. Salmè and S. Simula, Phys. Lett. B332, 1 (1994).

[20] S. R. Amendolia, G. Batignani, G. A. Beck, E.H. Bellamy, E. Bertolucci, G. Bologna, L. Bosito, C. Bradaschia, M. Budinich, M. Dell'Orso, B. D'Ettore Piazzoli, F.L. Fabbri, F. Fidecaro, L. Foa, E. Focardi, S.G.F. Frank, P. Gianetti, A. Giazzotto, M.A. Giorgi, M.G. Green, G.P. Heath, M.P.J. Landon, P. Laurelli, F. Liello, G. Mannocchi, P.V. March, P.S. Marrocchesi, A. Menzione, E. Meroni, P. Picchi, F. Ragusa, L. Ristori, L. Rolandi, A. Scribano, A. Stefani, D. Storey, J.A. Strong, R. Tenchini, G. Tonelli, G. Triggiani, W. Von Schlippe and A. Zallo, Phys. Lett. B178, 116 (1986).

[21] J. Volmer, D. Abbott, H. Anklin, C. Armstrong, J. arrington, K. Assamagan, S. Avery, O. K. Baker, H.P. Blok, C. Bochna, E.J. Brash, H. Breuer, N. Chant, J. Dunne, T. Eden, R. Ent, D. Gaskell, R. Gilman, K. Gustafsson, W. Hinton, G.M. Hu- ber, H. Jackson, M.K. Jones, C. Keppel, P.H. Kim, W. kim, A. Klein, D. Koltenuk, M. Liang, G.J. Lolos, A. Lung, D.J. Mack, D. MacKee, D. Meekins, J. Mitchel, H. Mkrcthyan, B. Muller, G. Niculescu, I. Niculescu, D. Pitz, D. Potterveld, L.M. Qin, J. Reinhold, I.K. Shin, S. Spepanyan, V. Tadevosyan, L.G. Tang, R.L.J. van der Meer, K. Vansyoc, D. Van Westrum, W. Vulcam, S. Wood, C. Yan, W.-X. Zhao and B. Zihlmann, Phys. Rev. Lett. 86, 1713 (2001).

[22] C. N. Brown, C.R. Canizares, W.E. Cooper, A.M. Eisner, G.J. Feldman, C.A. Lichtenstein, L. Litt, W. Lockeretz, V.B. Montana and F.M. Pipkin, Phys. Rev. D8, 92 (1973).

[23] C. J. Bebek, C.N. Brown, M. Herzlinger, S. Holmes, C.A. Lichtenstein, F.M. Pipkin and L.K. Sisterson, Phys. Rev. D9, 1229 (1974).

[24] C. J. Bebek, C.N. Brown, M. Herzlinger, S.D. Holmes, F.M. Pipkin, S. Raither and L.K. Sisterson, Phys. Rev. D13, 25 (1976).

[25] C. J. Bebek, C.N. Brown, S.D. Holmes, R.V. Kline, F.M. Pipkin, S. Raither and L.K. Sisterson, Phys. Rev. D17, 1693 (1978). 